УДК 537.61

\title{
Continual Approach in the Mean Field Theory of Incommensurate Magnetic States in the Frustrated Heisenberg Ferromagnet
}

\author{
Sergey N. Martynov \\ Vasiliy I. Tugarinov* \\ Kirensky Institute of Physics, SB RAS \\ Akademgorodok, 50/38, Krasnoyarsk, 660036 \\ Russia \\ Aleksandr S. Martynov \\ Faculty of Physics \\ Moscow State University \\ Moscow, 119991 \\ Russia
}

Received 20.08.2016, received in revised form 10.10.2016, accepted 15.11.2016

The algorithm of approximate solution has been developed for the differential equation describing the anharmonical change of the spin orientation angle in the model of ferromagnet with the exchange competition between nearest and next nearest magnetic neighbors and the easy axis exchange anisotropy. The obvious dependence of the angle velocity from angle and initial condition has been derived by expanding the first integral of the equation in the Taylor series in vicinity of initial condition.

Keywords: incommensurate magnetic structures, soliton lattice, continual approach. DOI: $10.17516 / 1997-1397-2017-10-1-55-59$.

The theoretical description of incommensurate magnetic structures (IMS) in antiferromagnetic dielectrics in the framework of Landau phenomenological theory of the phase transition was elaborated by Dzyaloshinskii [1]. For helimagnet with the Dzialoshinskii-Moria antisymmetrical exchange leading to a Lifshitz invariant in a free energy expansion (relativistic mechanism of forming IMS) the energy minimization for the solutions within the anisotropic plane is reduced to the solution of static sine-Gordon equation. The equation has the anharmonic solutions in the form of elliptical integrals describing the inhomogeneous helical structure with the step changing when moving along the helix vector - the so called soliton lattice [2]. For helimagnets with competing exchange interactions between the nearest and further magnetic neighbours (the exchange mechanism) it is necessary to take into consideration the second derivatives (and highter ones in the general case) of an order parameter and the energy minimization can not be reduced to an analiticaly integrable differential equation. The aim of the present work is to develop the algorithm of approximate solution for the equation describing the anharmonic change of the spin orientation angle in the easy axis ferromagnet with the exchange competition between the nearest and next nearest neighbors. We consider the magnetic structure with the spin orientation within the anisotropy plane (the flat anharmonic helix) and depends on the one coordinate.

*unonav@iph.krasn.ru

(C) Siberian Federal University. All rights reserved 
The Hamiltonian of the classical spins $\mathrm{S}=1$ with the ferromagnetic and antiferromagnetic exchanges between nearest and next nearest neighbors accordingly and the easy axis exchange anisotropy (XXZ-model) has a form

$$
H=J_{1} \sum_{i}\left(\mathbf{S}_{i} \mathbf{S}_{i+1}+\delta S_{i}^{z} S_{i+1}^{z}\right)+J_{2} \sum_{i}\left(\mathbf{S}_{i} \mathbf{S}_{i+2}+\delta S_{i}^{z} S_{i+2}^{z}\right), \quad J_{1}<0, \quad J_{2}>0, \quad \delta>0 .
$$

The same relative anisotropy in the both exchanges allows to reduce the number of model parameters and simply separate an effect of frustration and anisotropy on the IMS energy. The orientation of the spin $S_{i}$ in the plane with the easy axis z is determined by the total exchange field from neighbor spins $S_{i \pm 1}$ and $S_{i \pm 2}$. At $\mathrm{T}=0$ all spins have the equal length equal to saturation one. The local field on the site i normalized on the exchange $J_{1}$ has the components

$$
\begin{aligned}
h_{z} & =(1+\delta) h_{z}^{0}, \\
h_{z}^{0} & =\frac{1}{2}\left(\cos \theta_{i+1}+\cos \theta_{i-1}+R\left(\cos \theta_{i+2}+\cos \theta_{i-2}\right)\right), \\
h_{x} & =\frac{1}{2}\left(\sin \theta_{i+1}+\sin \theta_{i-1}+R\left(\sin \theta_{i+2}+\sin \theta_{i-2}\right)\right),
\end{aligned}
$$

where $h_{z}^{0}$ is the z-component of exchange field without anisotropy, $\theta$ is a polar angle and $R=$ $J_{2} / J_{1}<0$ is a frustration parameter. The transition to the continual description is carried out by the Taylor series expansion of the neighbor spins angles on the each site

$$
\theta_{i}=\theta, \quad \theta_{i \pm 1}=\theta \pm \Sigma_{11}+\Sigma_{12}, \quad \theta_{i \pm 2}=\theta \pm \Sigma_{21}+\Sigma_{22}
$$

where $\Sigma_{\alpha \beta}$ are the sums of odd and even derivatives of the variable $\theta$

$$
\begin{aligned}
& \Sigma_{11}=\sum_{n=1}^{\infty} \frac{\theta^{(2 n-1)}}{(2 n-1) !}, \quad \Sigma_{12}=\sum_{n=1}^{\infty} \frac{\theta^{(2 n)}}{(2 n) !} \\
& \Sigma_{21}=\sum_{n=1}^{\infty} \frac{2^{2 n-1} \theta^{(2 n-1)}}{(2 n-1) !}, \quad \Sigma_{22}=\sum_{n=1}^{\infty} \frac{2^{2 n} \theta^{(2 n)}}{(2 n) !} .
\end{aligned}
$$

After substitution (3) the components (2) and longitudinal field on the spin (an energy density on the single interval in coordinate space) takes the forms

$$
\begin{aligned}
& h_{z}^{0}=\cos \left(\theta+\Sigma_{12}\right) \cos \Sigma_{11}+R\left(\cos \left(\theta+\Sigma_{22}\right) \cos \Sigma_{21}\right), \\
& h_{x}=\sin \left(\theta+\Sigma_{12}\right) \cos \Sigma_{11}+R\left(\sin \left(\theta+\Sigma_{22}\right) \cos \Sigma_{21}\right) . \\
& h_{\|}=\epsilon=h_{z} \cos \theta+h_{x} \sin \theta=\delta h_{z}^{0} \cos \theta+\epsilon_{0},
\end{aligned}
$$

where $\epsilon_{0}=\cos \Sigma_{11} \cos \Sigma_{12}+R \cos \Sigma_{21} \cos \Sigma_{22}$ is an energy in the isotropic case $\delta=0$. An orientation of each spin is uniquely determined by the collinearity condition of spins and local fields from the neighbor spins [3]. The transverse field on the spin must be equal to zero

$$
\begin{aligned}
& h_{\perp}=h_{z} \sin \theta-h_{x} \cos \theta=\delta h_{z}^{0} \sin \theta-\Delta_{0} \equiv 0, \\
& \Delta_{0}=\cos \Sigma_{11} \sin \Sigma_{12}+R \cos \Sigma_{21} \sin \Sigma_{22} .
\end{aligned}
$$

Taking into account the collinearity constraint (6) the magnetic energy density takes a multiplicative form - the anisotropic and frustration components hold as product terms

$$
\epsilon=\frac{1+\delta}{1+\delta \sin ^{2} \theta} \cdot \epsilon_{0} .
$$


Rewriting the general equation (7) in the following form

$$
\frac{\cos \Sigma_{11} \sin \Sigma_{12}+R \cos \Sigma_{21} \sin \Sigma_{22}}{\cos \Sigma_{11} \cos \Sigma_{12}+R \cos \Sigma_{21} \cos \Sigma_{22}}=\frac{\delta \sin \theta \cos \theta}{1+\delta \sin ^{2} \theta}
$$

one can make a general conclusion that anharmonicity in the change of angle $\theta$ (the derivatives of the second order and higher) is appeared at $\delta>0$, takes a maximum value at $\theta=(2 n+1) \pi / 4$ and vanish at $\theta=n \pi / 2$.

Further solution of equation (6) will be carried out in the linear anharmonicity approximation $\left(\theta^{\prime \prime}<<1\right)$, neglecting the derivatives which are higher than second order. In this approach the Eq. (6) takes an autonomous form

$$
\theta^{\prime \prime} \cdot \frac{\cos \theta^{\prime}+4 R \cos 2 \theta^{\prime}}{\cos \theta^{\prime}+R \cos 2 \theta^{\prime}}=\frac{2 \delta \sin \theta \cos \theta}{1+\delta \sin ^{2} \theta},
$$

and at the substitution $z=\theta^{\prime 2} / 2$ is integrated by quadratures

$$
I\left(z, z_{0}\right)=\int_{z_{0}}^{z} \frac{C(z)}{\epsilon_{0}} d z=\ln \left(1+\delta \sin ^{2} \theta\right),
$$

where $C(z)=\cos \sqrt{2 z}+4 R \cos 2 \sqrt{2 z}, \quad \epsilon_{0}(z)=\cos \sqrt{2 z}+R \cos 2 \sqrt{2 z}$. The variable $\mathrm{z}$ range $\left\{z_{0}, z_{\max }\right\}$ in the interval $\theta \in\{0, \pi / 2\}$. Expanding the integral in the Taylor series in vicinity of $z_{0}$ and taking into account that $a_{0}=I\left(z=z_{0}\right)=0$ we obtain the series

$$
I\left(z, z_{0}\right)=\sum_{n=1}^{\infty} a_{n} \cdot \frac{\left(z-z_{0}\right)^{n}}{n !}, \quad a_{n}=\left(\frac{C(z)}{\epsilon_{0}}\right)_{z_{0}}^{(n-1)} .
$$

At linear anharmonicity keeping first two nonzero terms of series (10) (the quadratic approximation) we obtain the obvious dependence of the angle velocity from angle and initial condition $z_{0}$

$$
\begin{aligned}
& z=z_{0}-\frac{\epsilon_{0}(z)}{3 R \cdot K\left(z_{0}\right)\left(1+2 \cos ^{2} \sqrt{2 z_{0}}\right)} \times \\
& \quad \times\left(\sqrt{C^{2}\left(z_{0}\right)-6 R \cdot K\left(z_{0}\right)\left(1+2 \cos ^{2} \sqrt{2 z_{0}}\right) \cdot \ln \left(1+\delta \sin ^{2} \theta\right)}-C\left(z_{0}\right)\right),
\end{aligned}
$$

where $K\left(z_{0}\right)=\sin \left(\sqrt{2 z_{0}}\right) / \sqrt{2 z_{0}}$.

The energy of the quarter of period divided by the corresponding length is equal to the average energy of one spin

$$
E\left(z_{0}\right)=\frac{(1+\delta) \int_{0}^{\pi / 2} \frac{\epsilon_{0}(z)}{1+\delta \sin ^{2} \theta} \frac{d \theta}{\sqrt{2 z\left(\theta, z_{0}\right)}}}{\int_{0}^{\pi / 2} \frac{d \theta}{\sqrt{2 z\left(\theta, z_{0}\right)}}} .
$$

The ground state of the solutions is determined by an extreme of the function (12) (the maximum in our case, because the fields and energy density are normalized on the $J_{1}<0$ ). The corresponding initial condition $z_{0}=z_{\text {extr }}$ parametrizes the ground state solution for each set of the parameters $\delta$ and $\mathrm{R}$ and after substituting into Eq. (11) determines the functional dependence of $\mathrm{z}$ on the angle $\theta$ in the ground state. The solutions with increasing helix pitch upon changing the angle between the spin and easy axis $\theta$ from zero to $\pi / 2\left(\theta^{\prime \prime}>0\right)$ are exist 
when the condition $z_{0} \geqslant z_{\min }=(1+4 R) /(1+16 R) \geqslant 0$ is fulfilled. It is impossible for the IMS wave vector to tend to zero at the phase transition from incommensurate to commensurate state, which also follows from the phenomenological analysis based on the Landau theory [2]. It is means that the transition on the model parameters $\delta$ and $\mathrm{R}$ is accompanied by step-like change of the magnetic structure vector and hence is a first order phase transition.

The range of the $\mathrm{z}$ variation from $z_{\text {extr }}$ to $z_{\max }$ is increased with increasing $\delta$ and takes a maximal value on the phase boundary with collinear phases. The difference $z_{\text {max }}-z_{\text {extr }}$ takes the maximum value equal to 0.4 in the triple point $R=1 / 2, \delta \approx 1.34$ where the energy of the soliton phase is equal to the energies of ferromagnetic and "up-up-down-down" phases.

To assess the application of the quadratic approximation at the expansion of the first integral (10) one makes a numerical integration in the triple point and compares the result with the quadratic and cubic decomposition (10) (Fig. 1).

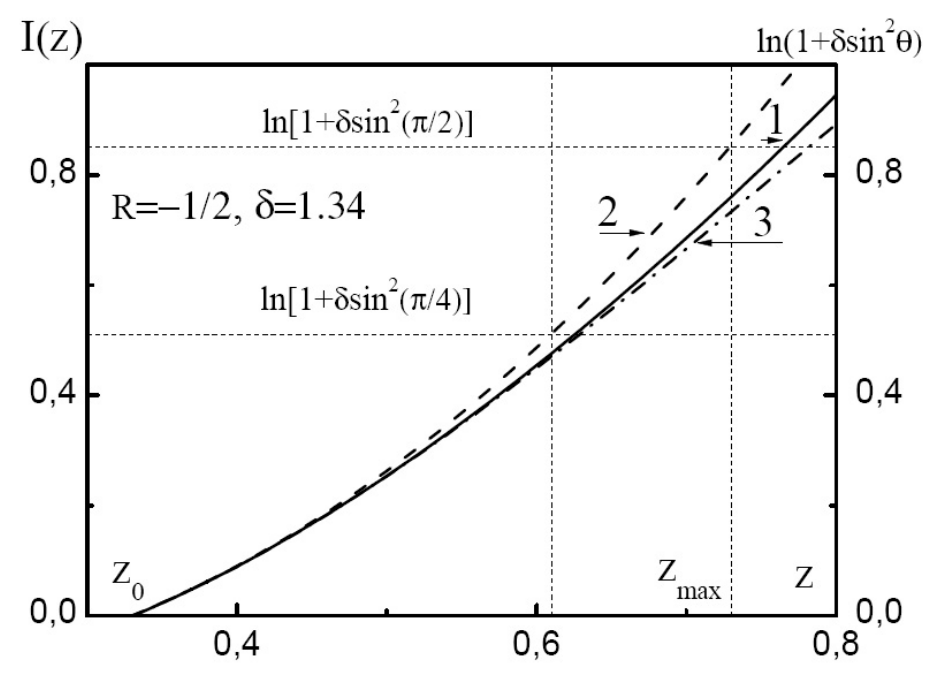

Fig. 1. The numerical integration of the first integral (9) (solid line 1) and the quadratic (dash line 2) and cubic (dash-dot line 3) approximations are shown with the right part of Eq. (9)

The coefficients $a_{1}$ and $a_{2}$ are positive. The third coefficient $a_{3}$ is negative and together with the next terms of expansion forms an alternate series which provides a fast expansion convergence to the numerical integration result.

\section{References}

[1] I.E.Dzyaloshinskii, Theory of helical structures in antiferromagnets. 1. Nonmetals, Sov. Phys. JETP, 19(1964), 960.

[2] Y.A.Izyumov, Neitronnaya diffraktsiya v dlinno-periodicheskih structurah (Neutron Diffraction in Long-Periodic Structures), Energoatomizdat, Moscow, 1987 (in Russian).

[3] S.N.Martynov, Flat and conical incommensurate magnetic structures in the two-subsystem partially frustrated heisenberg ferrimagnet, JMMM, 398(2016), 121. 


\section{Континуальное приближение в теории среднего поля несоизмеримых магнитных состояний во фрустрированном гейзенберговском ферромагнетике}

Сергей Н. Мартынов

Василий И. Тугаринов

Институт физики им. Л. В. Киренского СО РАН

Академгородок, 50/38, Красноярск, 660036

Александр С. Мартынов

Физический факультет

МГУ имени М.В.Ломоносова

Ленинские горы, 1/2, Москва, ГСП-1, 119991

Россия

Разработан алгоритм приближенного решения дифференциального уравнения, описывающего ангармоническое изменение угла ориентации спина в модели легкоосного ферромагнетика с конкурениией обменов между ближайшими и следующими за ближайшими магнитными соседями. Явная зависимость угловой скорости от угла и начального условия получена путем разложения первого интеграла уравнения в ряд Тейлора в окрестности начального условия.

Ключевые слова: несоизмеримые магнитные структуры, солитонная решетка, континуальное приближение. 